Egyptian

Orthodontic Journal

\title{
SUBJECTIVE APPRAISAL OF PATIENTS' OPINIONS OF FACTORS AFFECTING THEIR CHOICE FOR AN ORTHODONTIC PRACTICE
}

\author{
Sherif E Zahra ${ }^{1}$ and Tamer M Bedair ${ }^{1}$
}

ABSTRACT:

Objectives: The choice of an orthodontist and an orthodontic office sometimes is time consuming for patients and/or their parents. Some patients might do "shopping" for both before deciding on an appropriate one. This study was designed to identify factors affecting the choice of patients or their guardians when choosing a practice. Methods: All participants to take the questionnaire were 18 years and above and if under 18 their guardians were asked to fill in the questionnaire. The questions where presented both in English with a translation to "Arabic", the national language. The questionnaire consisted of 37 items covering various domains about the practitioner and his/her practice. The orthodontic offices were first contacted and those who accepted to participate in the study were paid a visit. For standardization, one of the authors was in charge of explaining the questionnaire to 6oth the orthodontist and the staff. Responses were given on a visual analog scale (VAS) Results: Respondents $(n=237)$ answered the questionnaire and females (66.1\%) were predominant over males (33.9\%). The Top scores were on items discussing the clinic in terms of "cleanness" followed equally by both "a clinic that operates on time" and "a clinic that uses the latest orthodontic technology and equipments". Conclusions: The orthodontic practice tidiness and being up-to-date and punctuality

1- Lecturer, Department of orthodontics, Faculty of dentistry, Suez Canal University, Egypt. 
Egyptian

Orthodontic Journal

seems to be the most important factor in patient's selection. Both novice and currently practicing orthodontists should be aware about the most important factors that patients or their guardians consider when they are making their minds and choosing an orthodontic practice.

\section{INTRODUCTION}

With increasing number of dental health providers, including orthodontists, it is very challenging to offer a better service and in the meantime, competition to have new prospective patients is higher. The most common channels for having new orthodontic customers are through referrals from dentists, staff referrals, advertising and insurance sources ${ }^{(1)}$. Patients reported back that to choose their orthodontist, they had to visit only one practitioner $59 \%$, some $25 \%$ visited two orthodontits and only $16 \%$ mentioned that have visited three or more offices ${ }^{(2)}$. This suggests that patients are very concerened about receiving a very refined service. They reported that their final selection of an orthodontic practice office was based mainly on caring attitude of the orthodontist $53 \%$, good reputation $49 \%$ and convenient office location $38 \%^{(2)}$

A prospective study ${ }^{(3)}$ was carried out, where orthodontists were asked to answer questions as their prospective patients would. There were two sets of questions, one for the prospective adult patients and the other for the parents of prospective adolescent patients. The questionnaire was to gather information on important characteristics, from patients' perspectives while choosing an orthodontic practice. Having a "caring attitude" and "A doctor who makes you feel comfortable" were the most important factors reported. The validity and reproducibility of the questionnaire used in the study was measured by the authors previously in a separate study ${ }^{(4)}$.

Retrospective studies ${ }^{(2,3,5)}$ carried out to measure patient preferences were generally conducted on patients who were already under or concluded their treatment. They are subject to memory bias and that's why gathering information while taking the decision strengthened our study and the conclusion accordingly ${ }^{(6)}$. 
Egyptian

Orthodontic Journal

This research was carried out for the purpose of providing both novice and currently practicing orthodontists with information about what are the most important factors that patients or their guardians consider when they are choosing an orthodontic practice. This in-turn might be useful in tailoring marketing strategies for the orthodontic office.

\section{SUBJECT AND METHODS}

The study protocol was granted approval from the review board and ethics committee at the faculty of dentistry, Suez Canal University. The research included independent participants (18 years or above) and dependent participants (below 18 years). If participants were under 18 years, parents were asked to fill in the questionnaire instead. Prospective patients were asked to quantify the importance of factors they felt important relative to the orthodontist and office while deciding about choosing their orthodontist. Exclusion criteria included any adult patient or their dependents if they have previously received orthodontic treatment or were currently under treatment.

The questions were presented in English and translated to Arabic by an office expert in medical science with the help of one of the authors (TB) for any professional orthodontic term.

The questionnaire used (Fig. 1) consisted of 37 items covering the following domains: the orthodontic office, the orthodontist (competency and personality), the staff and the financial arrangements. A question that was originally included under the domain of treatment facilities was dropped as insurance (governmental or private) doesn't cover orthodontic treatment.

Three questions were modified as follows: Question (Q29) where academic staff replaced board certification. Patients, generally consider the academic staff as they are more experienced. Question (Q3) was modified to "having a clinic that is functioning on Fridays" as it is a weekly holiday. Question (Q36) about the "Initial phone conversation with the receptionist" was modified to "effect of your first appointment in the clinic" as receptionists are not usually well trained and don't have the experience to answer questions in a professional manner. 
Some questions were not included from the original questionnaire as it is impossible to be applied such as offering a pick up and return from school for children to catch their appointments or arrangement of pool parties or movie nights for patients.

1-Having a clinic that is near your home

2-Having a clinic that is near to your workplace

3-Having a clinic that is open on Fridays

4-Having a clinic that is opened for evening appointments (after work)

5-Having a clinic that is opened for early morning appointments (before work)

6-Having a clinic that has a play area for little children

7-Having a modern looking clinic

8-Having a clinic that is clean

9-Having a clinic that has a television in the waiting area

10-having a clinic that uses the latest in the orthodontic technology and equipment

11-Having a clinic with refreshments in the waiting area (drinks/coffee/snacks)

12-Having a clinic that has information on a website

13-Having a clinic that has tooth-colored braces (ceramic)

14-Having a clinic that has invisible braces or aligners

15-Having a referral from your own dentist to visit this particular clinic

16-Having a recommendation from a family member/friend/acquaintance to visit this particular clinic

17-Having a clinic that displays photographs of previously treated patients

18-Having a doctor who wears a white coat

19-Having a doctor who has straight teeth

20-The age of the doctor

13-Having a clinic that has tooth-colored braces (ceramic)
21-The gender of the doctor

22-The doctor's religious beliefs

23-Having a doctor who makes you feel comfortable

24-Having a doctor who respects your feelings and with a caring attitude

25-Having a doctor who gives easy to understand explanations

26-The doctor's overall reputation

27-having a clinic that operates on time

28-Having a specialist with an orthodontic degree versus a general dentist who performs orthodontic treatment

29 -Having an orthodontist who is a staff in a dental faculty versus one who is not

30-Having a doctor who guarantees a shorter time in braces than a different doctor

31-Having the doctor's proposed treatment make sense to you

-Having a doctor whose treatment plan does not have teeth pulled for orthodontic treatment

33-Having a friendly clinic's staff with a caring attitude

34-Having a clinic with a good payment plan

35-Your total cost of treatment

36-Effect of your first appointment in the clinic

37-Having a doctor who does not require your child to wear headgear

Figure 1: Questionnaire for patients or their guardians 
Egyptian

Orthodontic Journal

The study was conducted in two phases. The first phase involved retrieving a list of all the registered orthodontists in the Egyptian orthodontic society and their offices' address. A demographic distribution of different practices (Hospitals and private orthodontic offices) and different locations that reflected the social and economic status of orthodontic customers were guaranteed. They were first contacted by phone and those who accepted to take part in the study were approached. Only one of the authors (SZ) was in charge of visiting and distributing the questionnaire to the private practioners' offices to ensure that the same message was delivered.

The second phase involved explaining briefly to both the orthodontist and front desk staff the questionnaire and instructions on how to answer it. For calibration, the staff were initially given a questionnaire to answer and any doubts were explained thoroughly.

For standardization, staff were asked to follow the next steps: introducing the questionnaire to new (first time) and recall patients (enrolled in treatment within one month) who do not mind participating in the study. Any queries/doubts should be cleared to respondents. The aim of the questionnaire has to be explained, how to answer the questions and putting a mark on the scale and help should be offered for those who need or have any doubts but not in a way to affect their choice. Time to answer questions was not an issue as pilot study for standardization showed that 20 minutes is the maximum time taken to answer the questionnaire.

Participants were asked to place a mark on a $100 \mathrm{~mm}$ line that correspond to the level of importance they believe it is. The scale of assessment was anchored on the left side $(0 \mathrm{~mm})$ with "not important at all" and on the right side $(100 \mathrm{~mm})$ with "most important".

Visual analog scale (VAS) has been proved as a valid and reliable measurement tool ${ }^{(7)}$. The participants or their guardians were asked to provide additional information about their demographic characteristics (age, gender, level of education and/or occupation).

Mean $( \pm 1 \mathrm{SD})$ response length on the VAS scale was calculated for all the questions for all participants in this study. Numerical data were 
Egyptian

Orthodontic Journal

presented as minimum, maximum, median, mean and standard deviation (SD) values. Qualitative data were presented as frequencies and percentages. We used frequencies for the description of the socio-demographic characteristics of the respondents. Statistical analysis was performed with IBM $^{\circledR}$ SPSS $^{\circledR}$ Statistics Version 20 for Windows.

\section{RESULTS}

A total of 237 surveys were collected from the various orthodontic offices with $94.5 \%$ (224 surveys) completely filled. When no response to a question affected the validity of data analysis, the incomplete survey was excluded. Respondents were predominantly females $66.1 \%$ and $33.9 \%$ were males. The mean \pm standard deviation values of age were $28.1 \pm 12.1$ years with a minimum of 16 years and a maximum of 40 years old. The majority were college graduates $(56 \%)$, parents of patients $(27 \%)$ and the rest $(17 \%)$ were still studying. Residents of urban areas represented the majority of our sample $(n=200,89.3 \%)$ compared to the residents of rural areas $(n=24,10.7 \%)$.

Questionnaire results are presented in Table (1) as median and mean scores. The 10 factors that respondents found most and least important are presented in Table (2). The highest scores were on items discussing the clinic in terms of cleanness (Q8) (mean=95.1) followed equally by both a clinic that operates on time (Q27) and a clinic that uses the latest orthodontic technology and equipments (Q10) (mean=94.4).

Both Items discussing the doctor "who makes you feel comfortable" (Q23) and "explains a treatment plan that makes sense to you" (Q31) came next with a Mean=92.9. Other factors as "easy to understand explanations" (Q25) (mean=92), “doctor's overall reputation" (Q26) (mean=91.6) and finally "who respects your feelings and with a caring attitude" (Q24) (mean=91.3) followed.

Finally the "effect of the first appointment" (Q36) and the "payment plan" (Q34) showed the least importance from the patient's perspective.

\footnotetext{
( IBM Corporation, NY, USA.

(R) SPSS, Inc., an IBM Company
} 
The least important factors reported were "Referrals from your own dentist" (Q10) followed by items concerned with the clinic " clinic near your home, clinic open on Fridays and a clinic with a play area for the children" (Q9, Q8 and Q6 respectively). Question (Q21) "The gender of the doctor" was of the least importance with a mean=25.9

When the 10 most important factors in the opinion of the male respondents $(n=76)$ were compared to the overall response (Table 2, Fig.2), the male respondents didn't include "having a clean clinic" and "a clinic that uses the latest technology" as their first two choices. They included instead (Q27) "having a clinic that operates on time" (mean $=94)$ and $(\mathrm{Q} 23)$ "a doctor who makes you feel comfortable" (mean=91.3). The only reported item by male respondents that was not mentioned in the overall response was " Having a modern looking clinic"(Q7) (mean=82.5) that was rated as last of the 10 most important factors that male respondents think about when they are choosing an orthodontic practice.

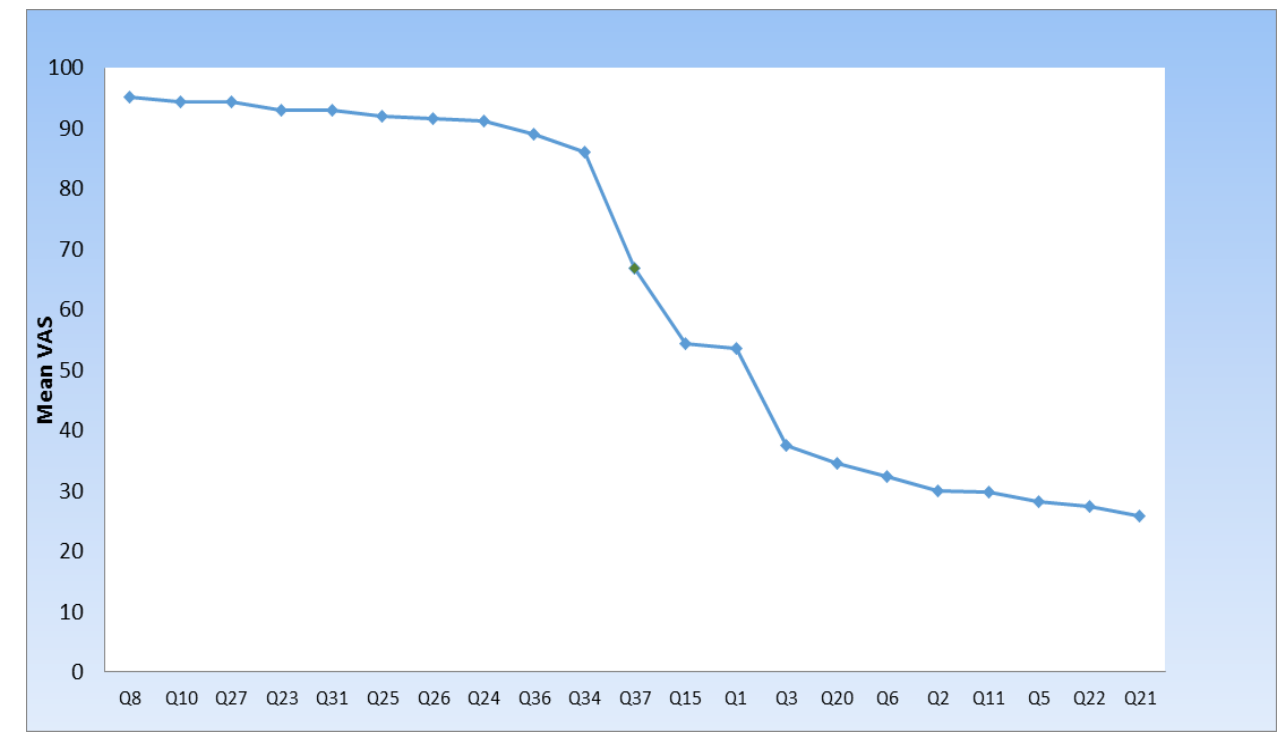

Figure 2: Mean VAS scores for the 10 most and least important factors in choosing orthodontic practice 
Egyptian

Orthodontic Journal

Table (2): Descriptive statistics of the 10 most and least important factors in choosing orthodontic practice

\begin{tabular}{|c|c|c|c|c|c|c|c|c|c|}
\hline & \multicolumn{3}{|c|}{$\begin{array}{l}\text { Whole sample } \\
(n=112)\end{array}$} & \multicolumn{3}{|c|}{$\begin{array}{c}\text { Males } \\
(n=38)\end{array}$} & \multicolumn{3}{|c|}{$\begin{array}{c}\text { Females } \\
(n=74)\end{array}$} \\
\hline & Question & Mean & $S D$ & Question & Mean & $S D$ & Question & Mean & $S D$ \\
\hline \multicolumn{10}{|l|}{ Top 10} \\
\hline 1 & Q8 & 95.1 & 13.6 & Q27 & 94.0 & 10.5 & Q8 & 97.7 & 4.9 \\
\hline 2 & Q10 & 94.4 & 13.5 & Q23 & 91.3 & 12.2 & Q10 & 97.5 & 4.4 \\
\hline 3 & Q27 & 94.4 & 10.3 & Q8 & 91.1 & 20.7 & Q31 & 95.2 & 7.8 \\
\hline 4 & Q23 & 92.9 & 11.4 & Q25 & 90.9 & 13.9 & Q27 & 95.1 & 9.0 \\
\hline 5 & Q31 & 92.9 & 11.1 & Q26 & 89.2 & 20.4 & Q24 & 94.1 & 10.2 \\
\hline 6 & Q25 & 92.0 & 14.5 & Q10 & 89.1 & 21.2 & Q23 & 93.9 & 10.7 \\
\hline 7 & Q26 & 91.6 & 14.7 & Q31 & 88.5 & 15.0 & Q26 & 92.8 & 11.0 \\
\hline 8 & Q24 & 91.3 & 17.4 & Q36 & 87.1 & 20.9 & Q25 & 92.5 & 14.9 \\
\hline 9 & Q36 & 89.1 & 18.3 & Q24 & 85.8 & 25.9 & Q34 & 91.4 & 11.0 \\
\hline 10 & Q34 & 86.0 & 20.6 & Q7 & 82.5 & 25.2 & Q33 & 91.1 & 12.3 \\
\hline $\begin{array}{l}\text { Middle } \\
\text { values }\end{array}$ & Q37 & 66.8 & 31.7 & & 61.1 & 31.8 & & 69.9 & 30.9 \\
\hline \multicolumn{10}{|c|}{ Bottom10 } \\
\hline 10 & Q15 & 54.3 & 31.3 & Q19 & 47.9 & 36.0 & Q1 & 55.8 & 36.9 \\
\hline 9 & Q1 & 53.5 & 37.6 & Q18 & 47.6 & 38.9 & Q15 & 52.9 & 31.0 \\
\hline 8 & Q3 & 37.5 & 36.7 & Q20 & 40.5 & 40.2 & Q3 & 37.8 & 38.6 \\
\hline 7 & Q20 & 34.6 & 35.9 & Q3 & 38.8 & 33.4 & Q11 & 33.7 & 30.2 \\
\hline 6 & Q6 & 32.3 & 34.3 & Q6 & 31.7 & 33.6 & Q6 & 32.9 & 35.0 \\
\hline 5 & Q2 & 30.1 & 32.5 & Q2 & 31.4 & 34.2 & Q20 & 31.8 & 34.0 \\
\hline 4 & Q11 & 29.9 & 28.8 & Q21 & 27.4 & 38.5 & Q22 & 29.8 & 35.7 \\
\hline 3 & Q5 & 28.2 & 33.0 & Q5 & 25.3 & 31.9 & Q2 & 29.6 & 32.0 \\
\hline 2 & Q22 & 27.5 & 35.6 & Q22 & 23.0 & 36.0 & Q5 & 29.4 & 33.8 \\
\hline 1 & Q21 & 25.9 & 35.0 & Q11 & 22.4 & 25.2 & Q21 & 25.2 & 33.6 \\
\hline
\end{tabular}

Volume 51 - June 2017 
Egyptian

Orthodontic Journal

When the 10 most important factors for the female respondents $(n=148)$ were compared with the overall response (Table 2, Fig.2), they included the same first two factors "clean clinic" and "clinic that uses the latest technology and equipments" (Q8 and Q10 respectively) as their first choices. The 10 most important responses for female respondents were in agreement with the overall pooled responses with different ranking (except for the first two).

One item "friendly clinic's staff with a caring attitude "(Q33) was not mentioned in the overall response and it came as their last option in the top 10 most important factors that will affect their choice for an orthodontic practice $($ mean $=91.1)$

\section{DISCUSSION}

The results displayed gives an overview for orthodontic practioners on factors that patients and/or their parents consider when they are deciding about choosing between orthodontic practices. It is reported that about $40 \%$ of patients generally do some "shopping" for an orthodontist ${ }^{(2)}$.

A structured questionnaire for prospective adult orthodontic patients and the parents of prospective child patients was used to evaluate the importance of the orthodontist, the office and the staff ${ }^{(4)}$. Some questions were modified to match the nature of patients where the questionnaire took place.

Patient satisfaction with both the orthodontist and the treatment is important to ensure compliance and adherence till end of treatment. In the present study, we found that the top three factors listed in final selection of an orthodontist are related to the clinic. A "clean orthodontic office" was listed as the top reason influencing respondents to select a specific orthodontic practice, closely followed by a one that "uses the latest orthodontic technology and equipment" and "operates on time". The variability of the society and their demands from one area to another when offered the choice of choosing the orthodontic practice entitled the importance of the clinic's environment as a factor affecting the opinion of the patients. This was different when compared to the factors that orthodontists thought they affect the patients' choice in another study ${ }^{(3)}$. The personal characteristics of the doctor and the staff were the most 
important factors found. The practitioner's reputation was most important along with the level of caring attitude the office projected ${ }^{(8)}$

Needless to say, if a payment plan is offered to patients and/or insurance coverage is available, this is an added advantage especially in areas with a high density of orthodontists and accordingly will be one of the driving considerations. This was clear in a survey ${ }^{(9)}$ carried where the service fees scored the highest driving factor. Closely scored were both the personality and the competency of the orthodontist, indicating that the fees is not the only main concern. Orthodontic treatment is a very expensive one and only afforded by upper moderate and higher income families in the middle-east and it is not listed in either governmental or private insurance. This explains why the respondents were attracted to office characteristics such as cleanness, excellence of the orthodontist, attention, and technology used while payment plan was not a crucial element during decision making.

The respondents to the survey were predominantly females. Their choices were in agreement with the overall responses of the sample. It is very important to consider the fact that females (mothers) are the population that take the decisions when selecting an orthodontic practice $(8,10)$. Also, it has been reported that girls seek orthodontic treatment more than boys ${ }^{(11)}$ and that females are more dissatisfied with the appearance of their dentition more than males ${ }^{(12)}$. This is different from previous studies where patients reported that the doctor's caring attitude was important to them and affected their choice ${ }^{(2,3,4)}$.

According to a study conducted by journal of clinical orthodontics on orthodontic practices ${ }^{(13)}$, referrals from general dentists is a corner stone to build up a new practice. In our survey, referrals from dentists $54.3 \%$ reported to be among the least ten factors that patients considered. This was in agreement with the overall response of the females as it came in their list with a low percentage $52.9 \%$. These findings stresses the importance of development of inter-personal and communication skills between dentists and orthodontists. Meanwhile, the providers of dental education, should make use of these findings to include classes on communication, leadership, and negotiation skills in their curricula, which proved to be successful in other settings $(14,15)$ 
The respondents did not feel that recommendation from a family member or a friend is highly appreciated to affect their decision (63\%). These findings were somewhat different from the traditional belief that personal information resources such as other dentists, friends and family are usually highly rated by respondents as an important influential factor $^{(16)}$.

On the other hand, a study reported that referrals from pediatric dentists accounted for $62 \%$ and $73 \%$ of them mentioned that it was an important factor to consider in their selection of an orthodontist ${ }^{(9)}$. The pediatric dentist is considered the main supplier of patients to orthodontists as he/she can recognize earlier changes during development of occlusion and hence refer the patients at the proper time. In our study, one possible explanation for this discrepancy is that in the middle-east, patients are still not aware enough about different specialties in dentistry and this explains why a referral is not considered an important factor and came with a low percentage.

Although the majority of the respondents were college graduates $56 \%$ and the demographic data showed that the level of education of parents is high, only $63 \%$ were interested in looking for an orthodontist's office over the web.

In another study ${ }^{(17)}$, the dental consumers not only used the web based information sources heavily, but also considered them to be highly important factor affecting their choice. The differences from our study could be attributed to the fact that either design of the home pages of some practices is either not attracting enough or lacks sufficient information and needs revision. This is an important factor to attract new patients searching for an orthodontist and to improve a practice's chance of making a good impression on prospects ${ }^{(18)}$. Online marketing including a modern website, online advertising and social media is essential if an orthodontist wants new patients to find his/her practice ${ }^{(19)}$. The other interpretation is that the population is not yet a web-savvy nor comfortable using computer for significant decisions. 
The attitude of patients towards dental treatment changes over time $^{(20)}$. It has been reported that with increasing demands on appearance and look, the number of patients seeking orthodontic treatment has increased. So it was important to address the demands on how to provide orthodontists with information on the factors affecting the choice of patients when choosing a practice. Postgraduate programs can make use of this survey to prepare residents what to provide and what patients expect in return to have a successful practice and to attract new patients according to their expectations.

Our study has some limitations. One limitation is the lack of generalizability from a nonstatistical purposive sampling. We chose this sampling to elicit a broad range of views, from which we can generate hypotheses for future statistical testing. The findings reflect only the factors affecting the choice of patients that seeks treatment in private practice. Therefore the results cannot be generalized to all patients seeking treatment whether in private or governmental facilities. Another limitation is that the age range of our sample is broad. Yet adolescent patients are not the one who decides about treatment and accordingly the choice of an orthodontic practice ${ }^{(21)}$.

This study was able to derive a structural pattern, which may help to conceptualize and integrate future approaches in the area of satisfaction of orthodontic patients. The data provided here may shed some light to strategically marketing orthodontic services, which in turn, will maximize number of patients. Qualitative research is needed to explore the trend of patient satisfaction with their treatment and accordingly, choice of practice to data obtained from this study about the factors that influenced their choice at the very beginning.

\section{CONCLUSIONS}

Many factors contribute to the selection of one orthodontist over another. Our study highlights some of the most important areas in which a practice can influence stake holders. The orthodontic practice tidiness and being up- to- date and punctuality seems to be the most important factor in patient's selection. 
Egyptian

Orthodontic Journal

\section{REFERENCES}

1- Bellavia D.C.J. Marketing your practice. J Clin Orthod. 1986 ;20:782-5.

2- Edwards, D.T, Shroff, B., Lindauer, S.J, Fowler, C.E. \& Tufekci, E. Media advertising effects on consumer perception of orthodontic treatment quality. Angle Orthod. 2008;78:771-7.

3- Bedair, T. M., Thompson, S., Gupta, C., Beck, F. M., \& Firestone, A. R. Orthodontists' opinions of factors affecting patients' choice of orthodontic practices. Am. J of Orthod and Dentofacial Orthop. 2010;138, 6.e1-6.e7.

4- St. Louis, B. L., Firestone, A. R., Johnston, W., Shanker, S., \& Vig, K. W. L. Prospective patients rate practice factors: Development of a questionnaire. Am.J. of Orthod and Dentofacial Orthop. 2011;139:235-241.

5- Walley EK, Silberman SL, Tuncay OC. Patient and parent preferences for orthodontic practices. Clin Orthod Res. 1992; 2:110-23.

6- Margetts, B., Vorster, H., Venter, C. Evidence-based nutrition: the impact of information and selection bias on the interpretation of individuals studies. South Afr J Clin Nutr 2003;16:78-87.

7- Gould, D., Kelly, D., Goldstone, L.,Gammon, J. Examining the validity of pressure ulcer risk assessment scales: developing and using illustrated patient simulations to collect the data. Journal of Clinical Nursing 2001;10: 697-706.

8- Walley, E.K, Silberman, S.L, Tuncay, O.C. Patient and parent prefrences for orthodontic practices. Clin Orthod Res. 1999;2:110-23.

9- Longoria, J.M, English, J., O'Neill, P.N, Tan, Q., et al. Factors involved in choosing an orthodontist in a competitive market. J. Clin. Orthod. 2011; 45:333.

10- Dorsey, J., Korabik, K. Social and psychological motivations for orthodontics treatment. Am. J. Orthod.1977;72:460.

11- O'Mullane, D.M., Robinson, M.E. The distribution of dentists and the uptake of dental treatment by schoolchildren in England.

Community Dent Oral Epidemiol.1977;5:156-9. 
Egyptian

Orthodontic Journal

12- Shaw, W.C. Factors influencing the desire for orthodontic treatment. Eur J Orthod. 1981;3:151-62.

13- Keim, R.G.,Gottlieb, E.L., Nelson, A.H., Vogels, D.S. orthodontic practice study, Part 3: practice growth and staff data. J. Clin. Orthod. 2009;43:625-634.

14- DePaola, D.P., Slavkin, H.C. Reforming dental health professions education: a white paper. J Dent Educ. 2004;68:1139-1150.

15- Roth, K. Dental education: a leadership challenge for dental educators and practitioners. J Dent Educ. 2007;71(8):983-987.

16- Book, D.S., Stockton H.J. Why patients choose a particular dentist. J Can Dent Assoc. 1986;52:123-6

17- Kim, M.J., Damiano, P.C., Hand, J. et al. Consumers' choice of dentists: how and why people choose dental school faculty members as their oral health care providers. J Dent Educ. 2012;76:695-704.

18- Wallin, W.S. Does your web site draw new patients? Am J Orthod Dentofacial Orthop. 2009;136:746-51.

19- Jorgensen, G . Attracting orthodontic patients via the Internet: A 20-year evolution. Am J Orthod Dentofacial Orthop. 2015;148:939-42.

20- Bos, A., Hoogstraten, J., Prahl-Andersen, B. A comparison of dental health care attitudes in the Netherlands in 1985, 1995, and 2001. Community Dent Oral Epidemiol. 2003;31:207-12.

21- Gosney, M.B. An investigation into some of the factors influencing the desire for orthodontic treatment. Br J Orthod. 1986;13:87-94. 